Istituto di Medicina Legale e delle Assicurazioni dell'Università di Roma

(Direttore: Prof. C. Gerin)

\title{
Prime osservazioni sulla distribuzione in Italia degli antigeni del sistema Kidd Ricerche sulla popolazione residente nel Lazio
}

\author{
Dott. Renato Giorda
}

Assistente

Nel r95 I'Allen, il Diamond ed il Niedziela dimostrarono che il siero di una donna - la quale dopo cinque gravidanze normali portate a termine senza essere mai stata sottoposta a terapia trasfusionale aveva successivamente partorito un neonato affetto da eritroblastosi - conteneva, oltre ad un anti-Kell, un'agglutinina irregolare, capace di riconoscere un antigene ignoto: antigene che venne definito " Kidd », dal cognome della malata.

L'anti-Kidd, così messo in evidenza, mostrava un titolo di $1 / 16$ sia in soluzione fisiologica, sia in mezzo albuminoideo, titolo crescente sino a $1 / 64$ con il test di Coombs. La temperatura ottimale per l'agglutinazione oscillava intorno ai $37^{\circ} \mathrm{C}$.

Attraverso ulteriori ricerche gli stessi Autori stabilirono che l'antigene Kidd era presente nel $77 \%$ degli individui di razza bianca di Boston e che si trasmetteva come un fattore mendeliano monomerico dominante; essi proposero di siglarlo con le lettere $\mathrm{Jk}^{\mathrm{a}}$. In seguito il Race, la Sanger, l'Allen, il Diamond ed il Niedziela formularono l'ipotetica esistenza di due geni, $\mathrm{Jk}^{\mathbf{a}}$ e $\mathrm{Jk}^{\mathbf{b}}$, dal primo dei quali essi ritenevano provenisse nell'emazia il fattore $\mathrm{Jk}^{\mathbf{a}}$. In effetti, qualche anno dopo (i953) la Plaut, la Ikin, il Mourant, la Sanger ed il Race identificarono l'anticorpo anti-Jk ${ }^{b}$, confermando pertanto la suddetta ipotesi.

In tal modo furono dunque ammessi due geni, $\mathrm{Jk}^{\mathrm{a}} \mathrm{e} . \mathrm{Jk}^{\mathrm{b}}$, coodominanti, che danno luogo alla formazione di tre diversi genotipi - con fenotipi omologhi - come è indicato nello schema che segue:

$$
\begin{array}{ll}
\mathrm{Jk}^{\mathrm{a}} & \mathrm{Jk}^{\mathrm{a}} \\
\mathrm{Jk}^{\mathrm{a}} & \mathrm{Jk}^{\mathrm{b}} \\
\mathrm{Jk}^{\mathrm{b}} & \mathrm{Jk}^{\mathrm{b}}
\end{array}
$$

Gli studi condotti in rapporto ad altri gruppi sanguigni dimostrano che il nuovo sistema è geneticamente indipendente da quelli già conosciuti.

Quanto alla distribuzione degli antigeni Kidd nei vari gruppi etnici, sono state registrate in merito sensibili differenze: ad esempio, nelle popolazioni negre della 
Nigeria e del Kenia la frequenza dell'antigene $\mathrm{Jk}^{\mathrm{a}}$ è pari al $95 \%$, notevolmente più elevata, cioè, di quella $(77 \%)$ della razza bianca (Ikin, Mourant).

Tenuto conto della mancanza di indagini del genere nel nostro Paese, riteniamo opportuno rendere note quelle da noi fino ad oggi effettuate su i io soggetti residenti nel Lazio. Precisiamo che per le ricerche medesime abbiamo potuto utilizzare soltanto il siero anti-Jka dell'Istituto Sieroterapico Berna - in quanto non è in commercio il siero anti-Jk $\mathrm{j}^{\mathrm{b}}$ - e che abbiamo dovuto limitarci all'esame di i ro soggetti perchè il siero anti-Jk $\mathrm{J}^{\mathbf{a}}$ è reperibile in quantità assai modeste.

È stata adottata la tecnica del test di Coombs indiretto, che qui succintamente riportiamo. Si prepara una sospensione salina al $2-5 \%$ degli eritrociti in esame; si pone quindi 0,1 cc di questa in una provetta e dopo aggiunta di o, I cc di siero test si lascia il tutto in incubazione a bagno-maria a $37^{\circ} \mathrm{C}$, per Io minuti.

I globuli rossi, in tal modo sensibilizzati, vengono poi accuratamente lavati per 4 volte e dopo l'ultimo lavaggio si addiziona soluzione fisiologica sino a ottenere una concentrazione al $50 \%$ di eritrociti; se ne allestisce successivamente una sospensione al $2 \%$. I globuli rossi si cimentano infine con il siero anti-globuline umane in parti eguali e si osserva la reazione, previa incubazione a $37^{\circ} \mathrm{C}$ per $3^{\circ}$ minuti.

La lettura è attuabile macroscopicamente, dopo avere leggermente agitato il sedimento, e controllabile microscopicamente ponendo una goccia del miscuglio su di un vetrino porta-oggetti e ricoprendola con un altro vetrino.

Nelle nostre indagini i risultati sono stati i seguenti:

$$
\begin{aligned}
& \mathrm{Jk}(\mathrm{a}+)=84 \\
& \mathrm{Jk}(\mathrm{a}-)=26
\end{aligned}
$$

La distribuzione per la popolazione residente nel Lazio - calcolata sui i п soggetti esaminati - è pertanto:

$$
\begin{aligned}
& \mathrm{Jk}(\mathrm{a}+)=0,7636(76,36 \%) \\
& \mathrm{Jk}(\mathrm{a}-)=0,2364(23,64 \%)
\end{aligned}
$$

Le frequenze geniche sono quindi calcolabili come in appresso:

$$
\begin{array}{ll}
\mathrm { Jk } ^ { \mathrm { b } } = 1 \longdiv { \mathrm { o } , 2 3 6 4 } & =0,4862 \\
\mathrm{Jk}^{\mathrm{a}}=\mathrm{I}-\mathrm{o}, 4862 & =0,5^{\mathrm{I}} 3^{8}
\end{array}
$$

Le frequenze dei genotipi risultano:

$$
\begin{aligned}
& \mathrm{Jk}^{\mathrm{a} J k^{\mathrm{a}}}=\left(0,5^{\mathrm{I}} 38\right)^{2}=0,2639 \\
& \mathrm{Jk}^{\mathrm{a} J k^{\mathrm{b}}}=(0,5 \mathrm{I} 38 \times 0,4862 \times 2)=0,4996 \\
& \mathrm{Jk}^{\mathrm{b} J k^{\mathrm{b}}}=\left(0,4^{862}\right)^{2}=0,2364
\end{aligned}
$$


In conclusione, gli antigeni del sistema Kidd e i relativi geni cooperano ad una più ampia conoscenza del patrimonio genico di una determinata popolazione. Dal punto di vista medico-legale, poi, essi assumono particolare importanza nel campo dell'ematologia forense, in quanto nelle indagini dirette all'accertamento del rapporto parentale riesce di grande interesse precisare il grado di probabilità del rapporto medesimo. Qualora, dunque, ai dati relativi alle proprietà degli altri sistemi gruppali già noti si associno quelli del sistema Kidd, si potrà pervenire ad un maggior grado di probabilità nel tentativo di riconoscimento del rapporto parentale: la ricerca medico-legalc in oggetto potrà acquistare così un valore giudiziario più probativo.

\section{Riassunto}

Dopo avere ricordato le principali fonti bibliografiche sull'argomento, l'Autore rende noti i risultati di una serie di ricerche dirette a stabilire la distribuzione degli antigeni del sistema Kidd nella popolazione residente nel Lazio. Tali ricerche hanno permesso di stabilire, su i io soggetti esaminati, l'esistenza delle seguenti percentuali:

$$
\mathrm{Jk}(\mathrm{a}+) 76,36 \% ; \mathrm{Jk}(\mathrm{a}-) 23,64 \%
$$

L'Autore, calcolate successivamente le relative frequenze geniche e genotipiche, conclude richiamando l'attenzione sulla importanza degli antigeni del sistema Kidd dal punto di vista medico-legale, ai fini delle indagini dirette all'accertamento del rapporto parentale.

\section{Bibliografia}

Ali.en, Diamonis and Niedziela, A new blood-group antigen. Nature, London, i67, 482 (1951).

Greenwalt, Sasaky and Sneath Joan, Haemolytic disease of the new born caused by anti-Jka. Vox Sanguinis, $1,157(1956)$.

Hart Mia and Loghem Van, A further example of anti-Jka. Vox Sanguinis, 3, 72 (1953).

Hunter Lois, Lewis Marion and Ghown, A further example of Kidd ( $\mathrm{Jk}^{\mathrm{a}}$ ) haemagglutinin. Nature, London, I68, $790(\mathrm{I} 95 \mathrm{I})$.

Loghem Van, Heifr Anne Margaretha, Hart Mia and Ribeiro Sanches, A serum containing anti-Jkb, anti-C and anti-M. Vox Sanguinis, 3, I I5 (1953).

Lundevall, The Kidd blood-group system. Acta pathologica microbiologica scandinavica, $3^{8}$, 39 (I956).

Milne, Wallace, Ikin Eltzabeth and Mourant, The Kidd (anti-Jka) haemagglutinin: a third example. Lancet, I, 627 (1953).

Plaut Gertrude, Ikin Elizabeth, Mourant, Sanger Ruth and Race, A new blood-group antibody, anti-Jk ${ }^{\mathrm{h}}$. Nature, London, I7 I, 43 I (1953).

Race and Sanger Ruth, Blood groups in man. Blackwell, Oxford (i958).

Race, Sanger Rutil, Allen, Diamond and Niedziela, Inheritance of the human blood-group antigen Jka. Nature, London, I68, 207 (I95I).

Rosenfield, Vogel, Gibbel, Natalie, Ohno Grace and Haber Gladys, Anti-Jka : three new examples of the isoantibody. Frequency of the factor in Caucasians, Negroes and Chinese of the New York City. American journal of clinical pathology, 23, 1222 (1953). 
Rosfnfield, Ley, Haber Gladys and Harris Jean, A further example of anti-Jk ${ }^{\mathrm{h}}$. American journal of clinical pathology, 24, 1282 (1954).

Rufrí, Les groupes sanguins chez l'homme. Masson, Paris (1953).

Sanger Ruth, Race, Rosenfield and Vogel, A serum containing anti-s and anti-Jkb. Vox Sanguinis, 3 , 7 I (I953).

Stratron, The value of fresh serum in the detection and use of anti-Jka antibody. Vox Sanguinis, I, I6o (I956). UNGER, A method for detecting $\mathrm{Rh}^{\circ}$ antibodies in extremely low titer. Journal of laboratory and clinical medicine, 37,825 (195I).

\section{RÉSUMÉ}

Après avoir rappelé les principales sources bibliographiques sur le thème, l'Auteur fait connaître les résultats d'une série de recherches tendant à fixer la distribution des antigènes du système Kidd dans la population demeurant dans le Latium. Ces recherches ont permis de déterminer, sur 110 sujets examinés, l'existence des pourcentages suivants: Jk $(\mathrm{a}+) 76,36 \%$; Jk (a-) $23,64 \%$.

L'Auteur, après avoir calculé successivement les relatives fréquences géniques et génotipiques, conclut attirant l'attention sur l'importance des antigènes du eystème Kidd du point de vue médico-légal, pour les recherches visant à la constation du rapport de parenté.

\section{SUMMARY}

After mentioning the main data of literature concerning the subject, the Author reports on the result of some research directed to establish the distribution of antigenes of the Kidd system in the population resident in the region of Latium.

This research has allowed to establish, in 110 individuals examined the presence of the following percentages: $\mathrm{Jk}(\mathrm{a}+) \quad 76.36 \%$; Jk (a-) $23.64 \%$.

The Author calculates further on the relative genic and genotypic incidence and finally calls the attention on the importance of the antigenes of the Kidd system from the forensicmedical point of view for the surveys directed to ascertain the ratio in relationship.

\section{ZUSAMMENFASSUNG}

Verf bringt zunächst die betr. Quellenangaben aus der Literatur und berichtet dann über eine Reihe von Untersuchungsergebnissen zur Feststellung der Verteilung der Antigene des Kidd-systems bei der in Latium ansässigen Bevölkerung.

Bei diesen Untersuchungen liessen sich bei 110 untersuchten Personen folgende Prozent- sätze fest stellen: Jk $(\mathrm{a}+) 76,36 \%$; Jk (a-) $23,64 \%$.

Es wurde darauf die Häufigkeit der entsprechenden Gen- und Genotypen berechnet, und zum Schluss macht Verf auf die Wichtigkeit der Antigene des Kidd-systems vom gerichtsärztlichen Standpunkt für die Forschungen zur Feststellung des Verwandschaftsverhältnisses aufmerksam. 